\title{
Long-run strategic advertising and short-run Bertrand competition
}

\author{
Reinoud Joosten*
}

April 21, 2011

\begin{abstract}
We model and analyze strategic interaction over time in a duopoly. Each period the firms independently and simultaneously take two sequential decisions. First, they decide whether or not to advertise, then they set prices for goods which are imperfect substitutes.

Not only the own, but also the other firm's past advertisement efforts affect the current 'sales potential' of each firm. How much of this potential materializes as immediate sales, depends on current advertisement decisions. If both firms advertise, 'sales potential' turns into demand, otherwise part of it 'evaporates' and does not materialize.

We determine feasible rewards and (subgame perfect) equilibria for the limiting average reward criterion. Uniqueness of equilibrium is by no means guaranteed, but Pareto efficiency may serve very well as a refinement criterion for wide ranges of the advertisement costs.
\end{abstract}

JEL-codes: C72, C73, L13, M31, M37.

Keywords: advertising, externalities, average rewards, equilibria.

\section{Introduction}

We study strategic interaction over time in a duopolistic market in which advertising causes different types of externalities in the time-dimension. For this purpose, we design a game with joint frequency dependent stage payoffs which allows us to incorporate rather complex relationships, and analyze it with modifications of techniques traditionally used for infinitely repeated games. Each period the agents are engaged in Bertrand competition in a strategic environment determined by their past advertising efforts and the current advertising decisions, both taken as known for the pricing decision. A strategy in this framework is a prescription, or 'game plan', for the entire

${ }^{*}$ FELab \& Department of Finance \& Accounting, University of Twente, POB 217, 7500 AE Enschede, The Netherlands. Email: r.a.m.g.joosten@utwente.nl. I thank participants in the EARIE conference in Ljubljana, 2009, and especially José Luis Maraga-González, for comments and criticism. 
infinite time horizon consisting of advertising and pricing decisions for which the intertemporal effects of advertising efforts should be considered.

Each period is split up in two decision moments. First, both firms independently and simultaneously decide to advertise or not. Next, observing these decisions they set their prices. If a firm decides to advertise, it pays a 'fee' at the beginning of the period for advertisement efforts during that period. Hence, for the Bertrand competition phase the advertisement costs can be regarded as sunk. We model this phase under the assumption that consumption in a given period does not depend on the periods before, as may very well be assumed for instance, for certain non-durable or perishable goods.

Advertising has two effects separated in the time dimension, ${ }^{1}$ it affects immediate sales directly and future sales in a cumulative manner (cf., e.g., Friedman [1983]) and we aim to capture both. With respect to the direct, i.e., immediate or short-run, effects, advertising increases the own immediate sales given the action of the other firm. Advertising may also cause immediate externalities. Friedman [1983] distinguishes predatory and cooperative advertising. An increase in advertising efforts of one firm leads to a sales decrease of the other in the former type, to an increase in the second type of advertising. In our model we incorporated features to allow representation of both aspects concerning the short term effects of advertising.

There is also an indirect, or cumulative, effect of current advertisement on future sales. To capture this feature, we introduce the notion of sales potential varying in time under the decisions the firms have taken concerning their past advertising efforts (see also Joosten [2009]). The current sales potential of each firm is determined by its own but also by its opponent's past efforts. A higher effort of either firm leads to an increase of the sales potentials (ceteris paribus), but the impact of the own past efforts on the own potential is always stronger than the impact of the opponent's past efforts. Advertising is therefore cooperative in its cumulative effects on the sales potentials. ${ }^{2}$ How much of these materialize as immediate sales, depends on the current advertisement decisions.

The shapes of the current demand functions are determined 'roughly' by past advertising, whereas present efforts fix the demand functions in detail. To be more specific, high past cumulative advertisement expenditures shift the sales potential curves upward, high individual past expenditure make the own demand less price elastic; asymmetric efforts lead to a tilt of the same curves, making the more active firm less vulnerable to a price decrease of the less active one. Current demand functions are then fixed by the advertisement actions in the first decision moment of the stage and unless

\footnotetext{
${ }^{1}$ See also, e.g., Cellini \& Lambertini [2003], Lambertini [2005], Cellini et al. [2008].

${ }^{2}$ José Luis Maraga-González advised us to emphasize here that the basic model is general enough to deal with other scenarios as well.
} 
both firms advertise a part of the potential is lost ceteris paribus.

In our dynamic deterministic duopolistic leaderless model of Bertrand competition for non-durable incomplete substitutes, we restrict ourselves to persuasive advertising with long and short run externalities on sales. ${ }^{3}$ We assume that the firms wish to maximize the average profits over an infinite time-horizon. We determine equilibria employing modifications of techniques traditionally used to analyze infinitely repeated games. We find, as in the Folk Theorem for repeated games, that a continuum of rewards may exist which can be supported by an equilibrium involving 'threats'. Pareto efficiency seems a very useful criterion to reduce the abundance of equilibria from this Folk Theorem, as for many parameter constellations, there exists a unique Pareto efficient equilibrium.

The critical reader may wonder why one would desire an additional model given the affluence of advertising models. A major forte of our approach is its generality as we only require continuity of the functions involved. Empirical research should provide us with appropriate classes of dynamics and in that sense we are ready to deal with almost anything that empirics will throw at us. Furthermore, most empirical research takes measurements at certain predetermined points in time. Since our methods deal with decisions, actions and changes in discrete time, a translation effort into continuous time for instance is quite unnecessary. An additional advantage of our type of modeling is that it may serve as a vehicle of communication to large communities of economically inclined users who are not experts in the mathematical discipline of optimal control theory or dynamic programming, the dominant modes of analysis in differential and difference games. ${ }^{4}$

In an additional 'introductory' section, we relate our model to others in the literature. Next, we proceed with a section to introduce the advertisement model. Section 4 deals with strategies and rewards; Section 5 treats threats and equilibria. Section 6 concludes.

\section{Literature and the positioning of our model}

As mentioned, the games to be considered are games with joint frequency dependent stage payoffs, JFD-games for short, which generalize games with frequency dependent stage payoffs, or FD-games, introduced by Brenner \& Witt [2003], fully classified and analyzed in Joosten, Brenner \& Witt [2003]. Such games can be classified as stochastic games or difference games but we are apprehensive to do so, because both classes of games are closely associated to modeling traditions which are quite technically advanced and involved. ${ }^{5}$ JFD-games however, have the advantage that certain intertemporal

\footnotetext{
${ }^{3}$ If these terms are vague, please consult the literature section.

${ }^{4}$ Not to mention the didactic possibilities of our models.

${ }^{5}$ Stochastic games were introduced by Shapley [1953]. Amir [2003] shows interesting connections between difference games and stochastic games in economic applications. The
} 
externalities can be modeled conveniently. We gained experience investigating a range of problems in which current decisions influence payoffs or other relevant options in the future. We challenge(d) the virtual monopoly of differential games in models on advertising (Joosten [2009]), the exploitation of common (renewable) resource systems (Joosten [2007a,b,c,2010]), environmental pollution (Joosten et al. [2003], Joosten [2004]), changing preferences (Joosten et al. [2003]), and learning-by-doing (Joosten et al. [2003]).

Dorfman \& Steiner [1954] examine the effects of advertising in a static monopoly and derive necessary conditions for the optimal level of advertising. In a dynamic monopolistic model, Nerlove \& Arrow [1962] treat advertisement expenditures similar to investments in a durable good. This durable good is called goodwill which is assumed to influence current sales. Historical investments in advertisement increase the stock of goodwill, but simultaneously goodwill depreciates over time. Nerlove and Arrow derive necessary conditions for optimal advertising, thus generalizing the Dorfman and Steiner result. Friedman [1983] in turn generalizes the Nerlove-Arrow model to allow oligopolistic competition in advertising and derives necessary conditions for the existence of a noncooperative equilibrium (Nash [1951]).

Our notion of sales potential is quite close to goodwill in e.g., Nerlove \& Arrow [1962] and Friedman [1983]. The modeling of the changes in time in the former model follows the work of Vidale \& Wolfe [1957], though the authors quote Waugh [1959] as a main source of inspiration. Vidale \& Wolfe [1957] present an interesting field study giving empirical evidence of the positive effects of past advertising on current sales. Furthermore, once advertising expenditures are stopped, current sales do not collapse, but slowly deteriorate over time. Though Friedman quotes the work of Nerlove and Arrow as a source for the notion of goodwill, his technical treatment of the changes of the level of goodwill in time is inspired by Prescott [1973].

Economics has produced a large body of work on advertising. One source of variety is the modeling of time-related aspects. For instance, is the model static (e.g., Dorfman \& Steiner [1954]), or is it dynamic in the sense that the strategic environment may change (e.g., Nerlove \& Arrow [1962])? Another source of variety is the market under consideration, e.g., monopoly (Nerlove \& Arrow [1962]), oligopoly (Friedman [1983]), leaderfollower oligopoly (Kydland [1977]). A third one is possible combinations of advertising with other marketing instruments, e.g., Schmalensee [1978] combines advertising and quality. A fourth is the entity to be influenced by advertising, for instance sales (e.g., Nerlove \& Arrow [1962]) or market shares (e.g., Fershtman [1984]). A fifth is the strategic dimension in which competition on the market is chosen, for instance Cournot (e.g., Joosten

origin of difference games is not known to this author, but an influential example is the model of Levhari \& Mirman [1980] on common pool resource exploitation. Continuous time variants of difference games are differential games (see e.g., Dockner et al. [2000], Jørgensen \& Zaccour [2004], Engwerda [2005]). 
[2009]) or Bertrand competition (e.g., Cellini et al. [2008]).

Another dimension is based on the distinction by Nelson [1970] between search and experience goods. The characteristics of the former kind are known by-and-large before purchase, whereas those of the latter can be determined only after purchase. Advertising differs for the two types of goods because the information conveyed to the consumers differs. Informative advertising provides information on e.g., the price, availability or characteristics of a product; persuasive advertising tries to generate consumer interest for a product, often by association or through rather indirect 'channels'. An example of persuasive advertising would be a famous athlete shown drinking a certain beverage, or eating some kind of cereal. Credence goods (Darby \& Karni [1973]) can be regarded as an extreme type of experience good, as it is hard to determine their characteristics even after purchase. The quality of a certain brand of toothpaste can only be determined in the very long run after a visit to a dentist. Informative advertising is directed at search goods, persuasive advertising aims at experience or credence goods.

For dynamic optimal control models of advertisement Sethi [1977] performed a Herculean task by coming up with a classification distinguishing four types. The task may prove to be Sisyphean, as a more recent survey by Feichtinger et al. [1994] already features six classes. Three new categories were introduced, categories present in the earlier classification were renamed and expanded, and merely one category survived in its original form. The reader interested in differential games on advertising is referred to Dockner et al. [2000] and Jørgensen \& Zaccour [2004].

\section{The rules of the game}

The advertisement game is played by two firms (players) $A$ and $B$ at discrete moments in time called stages. Each stage the players independently and simultaneously take two decisions sequentially. First the firms decide whether to advertise or not; then, the firms set their prices knowing the advertisement decisions taken. The pricing decisions are assumed sufficiently independent that there are no forward or backward consumption externalities in the time dimension. We have two types of externality effects from advertising, an immediate one and one which develops gradually in time. We start by describing the Bertrand competition at a given stage assuming past and current advertisement decisions to be known. Next, we treat immediate externalities from advertising in the same stage, then we formalize the externalities over time, and finally connect these effects. 


\subsection{A Bertrand stage game}

We assume that at a given stage the two firms have the following demand functions:

$$
x_{k}=D_{k}-a_{1 k} p_{k}+a_{2 k} p_{\neg k},
$$

where $k=A, B$, and $\neg k$ denotes 'not $k$ '. So, for instance, firm $A$ 's demand $x_{A}$ depends on the level of its own price $p_{A}$, but also on $p_{B}$, the price set by the other firm. We take all parameters in (1) to be positive. This means that the demand for firm $A$ goes down if $A$ increases its price, but goes up if firm $B$ increases its price. This implies that the goods produced by both firms can be regarded as substitutes. The price spill-over effects captured by $a_{2 k}$, $k=A, B$, can not increase without bounds relative to the own price effects $a_{1 k}, k=A, B$, in this model. A condition which suffices for the ensuing analysis to make sense (technically speaking) is

$$
a_{1 k}>\frac{1}{2} a_{2 k} \text { for } k=A, B \text {. }
$$

It should be noted however, that we can make do with a much weaker condition which depends however, on the mutual spill-over effects.

The agents wish to maximize their stage payoffs using prices as strategic variables while facing linear costs, i.e., we have costs equal to

$$
c_{k}\left(x_{k}\right)=c_{k} x_{k}+c_{0, k} \text { for } k=A, B .
$$

with $c_{k}>0$ and $c_{0, k} \geq 0$ for $k=A, B$. Here, $c_{0, k}$ denotes the fixed costs, and $c_{k}$ the constant marginal costs for firm $k$.

The above means that the firms face the following rather standard maximization problems in the framework of Bertrand competition:

$$
\max _{p_{k}} p_{k} x_{k}-c_{k} x_{k}-c_{0, k} \text { for } k=A, B
$$

For the sake of easy reference, we introduce a notation for the parameter sets defining the demand functions, i.e., $(D, a)=\left(D_{A}, D_{B}, a_{1 A}, a_{2 A}, a_{1 B}, a_{2 B}\right)$.

Lemma 1 The Bertrand equilibrium $\left(p_{A}^{*}(D, a), p_{B}^{*}(D, a)\right)$ in the model determined by Eq. (1)-(4) is given by

$$
p_{k}^{*}(D, a)=\frac{2 a_{1 \neg k}\left(D_{k}+c_{k} a_{1 k}\right)+a_{2 k}\left(D_{\neg k}+c_{\neg k} a_{1 \neg k}\right)}{4 a_{1 A} a_{1 B}-a_{2 A} a_{2 B}} \text { for } k=A, B .
$$

The profit maximizing quantity sold for firm $k=A, B$, is then given by

$$
x_{k}^{*}(D, a)=\frac{1}{2}\left(D_{k}-c_{k} a_{1 k}\right)+\frac{a_{1 k} a_{2 k}\left(D_{\neg k}+c_{\neg k} a_{1 \neg k}\right)}{4 a_{1 A} a_{1 B}-a_{2 A} a_{2 B}} .
$$




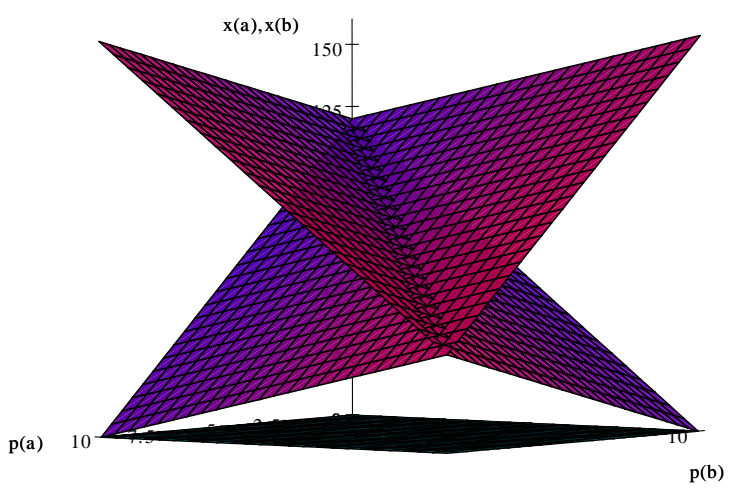

Figure 1: Demand curves for both firms in Example 1 as an illustration of Eq. (1). If both prices (denoted here by $p(a)$ and $p(b)$ ) are zero, demand is equal to 120, if firm A sets a price of 10, then its demand ranges from 0 to 40, whereas B's demand ranges from 160 to 40, both for increasing $p(b)$.

Finally, we can write down the profits for both players depending on the parameters of the demand functions.

$$
\pi_{k}^{*}(D, a)=p_{k}^{*}(D, a) x_{k}^{*}(D, a)-c_{k}\left(x_{k}^{*}(D, a)\right), k=A, B .
$$

We will now give a fairly standard numerical example to compare it with our expansions in the sequel.

Example 1 Let for $k=A, B$, the demand and the cost curves be given by

$$
\begin{aligned}
x_{k} & =120-12 p_{k}+4 p_{\neg k}, \\
c_{k}\left(x_{k}\right) & =3 x_{k}+200 .
\end{aligned}
$$

Here, $x_{k}$ denotes the demand for firm $k$, given its own price $p_{k}$ and the price of its competitor $p_{\neg k}$. These demand curves are illustrated in Figure 1.

From Eq. (5) we derive the following specifics for the reaction curves.

$$
p_{k}\left(p_{\neg k}\right)=\frac{156+4 p_{\neg k}}{24} .
$$

Figure 2 provides an illustration of these reaction curves. Lemma 1 yields the Bertrand equilibrium:

$$
\left(p_{A}^{*}, p_{B}^{*}\right)=(7.8,7.8)
$$


This can be conveniently confirmed in Figure 2, as the Bertrand equilibrium coincides with the intersection point of the reaction curves. The associated (profit maximizing) sales are then given by

$$
x_{A}^{*}=x_{B}^{*}=55.371 .
$$

The associated profit levels can be computed as being equal to

$$
\pi_{A}^{*}=\pi_{B}^{*}=65.783 .
$$

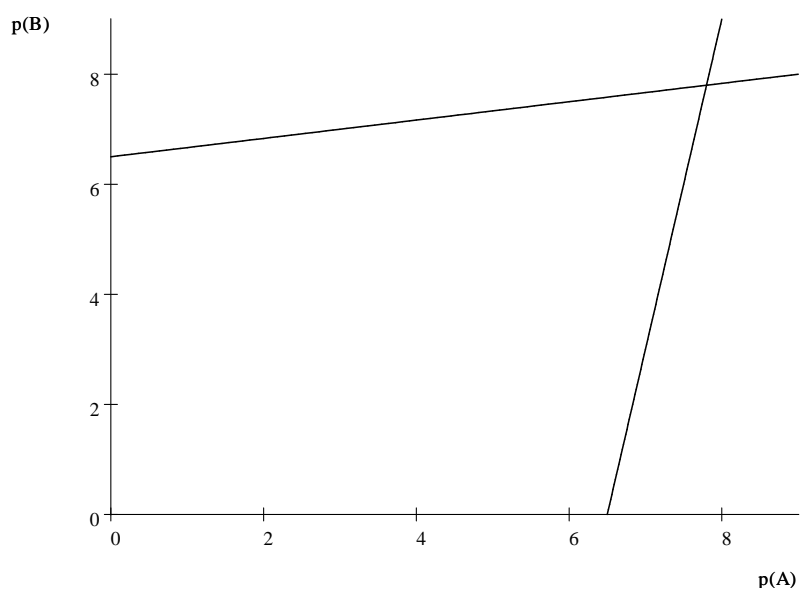

Figure 2: The reaction curves in the price dimension for Example 1. The intersection point is to be associated with a Bertrand equilibrium, i.e., prices are mutual best-replies.

\subsection{Immediate effects of advertising}

Now, we assume that there is an immediate effect of advertising on the demand functions of both firms. We choose the following notations

$$
x_{k}^{j^{A}, j^{B}}=D_{k}^{j^{A}, j^{B}}-a_{1 k}^{j^{A}, j^{B}} p_{k}+a_{2 k}^{j^{A}, j^{B}} p_{\neg k} .
$$

where $j^{k} \in\{1,2\}$ denotes the action regarding advertising chosen by player $k \in\{A, B\}$ in the same stage. So, there are four possible action combinations depending on the advertisement decisions of the agents at the beginning of the stage. We do not assume that the costs of production depend on the advertising decisions of the agents, however, advertisement costs are not assumed zero. Stated differently, advertising decisions $\left(j^{A}, j^{B}\right)$ induce

$$
(D, a)^{j^{A}, j^{B}}=\left(D_{A}^{j^{A}, j^{B}}, D_{B}^{j^{A}, j^{B}}, a_{1 A}^{j^{A}, j^{B}}, a_{2 A}^{j^{A}, j^{B}}, a_{1 B}^{j^{A}, j^{B}}, a_{2 B}^{j^{A}, j^{B}}\right) .
$$




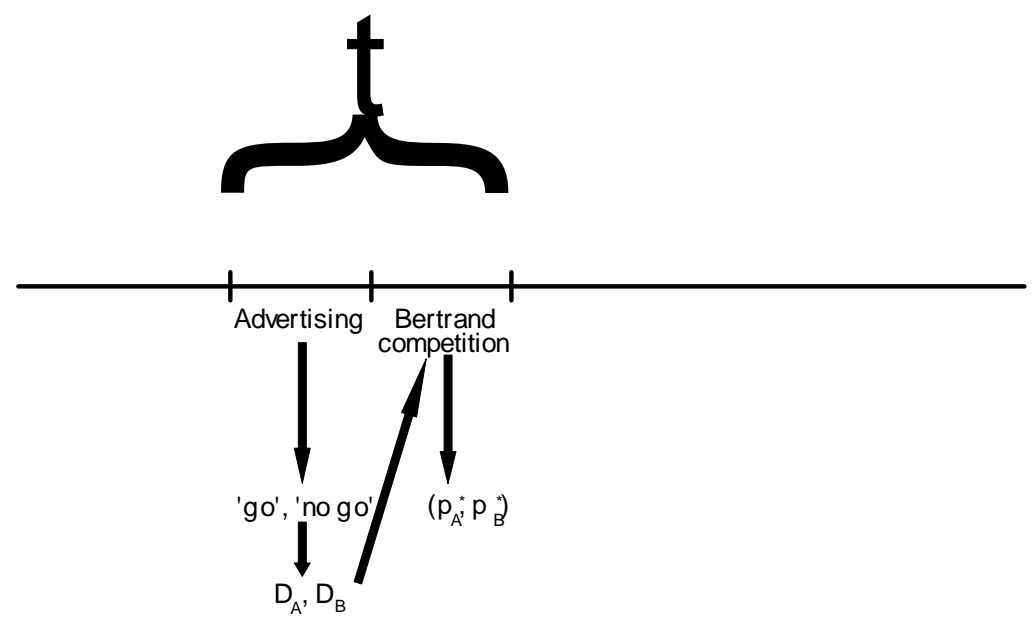

Figure 3: Timing each period. First the advertisement decisions are taken, then the pricing decisions. For the latter phase the outcome of the first is known and the choices influence the demand curves.

See Figure 3 for a graphic illustration of the timing issues involved.

For the sake of simplicity we assume that the shape of the demand curves does not change qualitatively. More specifically, the advertising decisions shift these curves up or down without changing their slope. Hence, to make sense in the present context we find the following specifications rather helpful. We assume that the relation between the various demand functions under the advertising decisions is such that $\gamma_{k}^{1,1} \geq \gamma_{k}^{2,1}, \gamma_{k}^{1,2} \geq \gamma_{k}^{2,2}>0$ exist satisfying

$$
\frac{x_{k}^{j^{A}, j^{B}}}{\gamma_{k}^{j^{A}, j^{B}}}=\frac{x_{k}^{\widetilde{j}^{A}, \widetilde{j}^{B}}}{{\widetilde{\gamma_{k}^{A}}}^{A} \widetilde{j}^{B}} \text { for } k=A, B, j^{A}, j^{B} \widetilde{j}^{A}, \widetilde{j}^{B} \in\{1.2\} .
$$

So, this will bring about the desired technical consequences with respect to the demand functions. We would like to highlight the following convenient property to be used in the remainder.

Lemma 2 Let $(D, a)=\left(D_{A}, D_{B}, a_{1 A}, a_{2 A}, a_{1 B}, a_{2 B}\right)$ be given, let $4 a_{1 A} a_{1 B}-$ $a_{2 A} a_{2 B}>0$ and $\left(D^{\prime}, a^{\prime}\right)=\left(\gamma_{A} D_{A}, \gamma_{B} D_{B}, \gamma_{A} a_{1 A}, \gamma_{A} a_{2 A}, \gamma_{B} a_{1 B}, \gamma_{B} a_{2 B}\right)$. Then $p_{k}^{*}\left(D^{\prime}, a^{\prime}\right)=p_{k}^{*}(D, a)$ and $x_{k}^{*}\left(D^{\prime}, a^{\prime}\right)=\gamma_{k} x_{k}^{*}(D, a)$.

The lemma implies for the setting formulated the following. 
Corollary 1 Under the assumption formulated in Equation (4), Lemma 2 implies

$$
p_{k}^{*}(D, a)^{j^{A}, j^{B}}=p_{k}^{*}(D, a)_{k}^{\widetilde{j}^{A}, \widetilde{j}^{B}} .
$$

So, under all different specifications the Bertrand equilibrium prices are identical. Hence, the optimal quantities and profit levels can be determined easily. Given advertising decisions $\left(j^{A}, j^{B}\right)$, advertisement costs $A C^{A}, A C^{B}$, we have the following matrix game at the beginning of the stage

$$
\left[\begin{array}{cc}
\pi_{A}^{1,1, *}-A C^{A}, \pi_{B}^{1,1, *}-A C^{B} & \pi_{A}^{1,2, *}-A C^{A}, \pi_{B}^{1,2, *} \\
\pi_{A}^{2,1, *}, \pi_{B}^{2,1, *}-A C^{B} & \pi_{A}^{2,2, *}, \pi_{B}^{2,2, *}
\end{array}\right],
$$

where, for instance, $\pi_{A}^{1,1, *}$ is a short-hand notation for $\pi_{A}^{*}\left((D, a)^{1,1}\right)$.

Example 2 Advertising is not for free and it is assumed that the advertisement costs are fixed and equal for both firms, i.e.,

$$
A C^{A}=A C^{B}=200 .
$$

We continue the example given before in the sense that we assume the demand functions if neither firm advertises to be given by

$$
x_{k}^{2,2}=120-12 p_{k}+4 p_{\neg k}, k=A, B .
$$

With respect to the immediate effects of advertising we assume that

$$
\begin{aligned}
& x_{k}^{1,1}=2 x_{k}^{2,2} \quad \text { for } k=A, B \\
& x_{A}^{1,2}=\frac{7}{4} x_{A}^{2,2} \quad x_{B}^{1,2}=\frac{5}{4} x_{B}^{2,2} \\
& x_{A}^{2,1}=\frac{5}{4} x_{A}^{2,2}
\end{aligned}
$$

It can be confirmed readily that for given price levels, total sales increase if advertising efforts in the industry increase, i.e.,

$$
x_{A}^{1,1}+x_{B}^{1,1}>x_{A}^{1,2}+x_{B}^{1,2}=x_{A}^{2,1}+x_{B}^{2,1}>x_{A}^{2,2}+x_{B}^{2,2},
$$

where we omit the notations for the price levels. Moreover,

$$
x_{A}^{1,1}>x_{A}^{1,2}>x_{A}^{2,1}>x_{A}^{2,2} \text { and } x_{B}^{1,1}>x_{B}^{2,1}>x_{B}^{1,2}>x_{B}^{2,2},
$$

again ceteris paribus. Hence, own sales increase in own marketing efforts but also in the marketing efforts of the competitor. In this sense advertising in this example partially has a public good character (Fershtman [1984]). In other words, there exists the following advertising externality: keeping one player's action fixed, advertising of his opponent increases the former's 
sales as well. There are however, differences as to which firm benefits with respect to sales more from advertising for 'asymmetric' advertising, i.e.,

$$
x_{A}^{1,2}>x_{B}^{1,2} \text { and } x_{B}^{2,1}>x_{A}^{2,1} .
$$

This means that the firm engaging in advertising has higher sales than the inactive firm.

Lemma 2 allows us to write down the associated payoff matrix easily as:

$$
\begin{aligned}
& {\left[\begin{array}{cc}
\pi_{A}^{1,1, *}-A C^{A}, \pi_{B}^{1,1, *}-A C^{B} & \pi_{A}^{1,2, *}-A C^{A}, \pi_{B}^{1,2, *} \\
\pi_{A}^{2,1, *}, \pi_{B}^{2,1, *}-A C^{B} & \pi_{A}^{2,2, *}, \pi_{B}^{2,2, *}
\end{array}\right] } \\
= & {\left[\begin{array}{cc}
331.56,331.56 & 265.12,332.23 \\
332.23,265.12 & 65.78,65.78
\end{array}\right] }
\end{aligned}
$$

Note that the stage game has two pure Nash equilibria, namely top-right and bottom-left, hence in equilibrium precisely one firm advertises. The firm advertising appropriates a greater share of the sales increase, but bears all costs; the other firm benefits moderately from its competitor's efforts absolutely for free. There is also a mixed Nash equilibrium in the stage game, Pareto dominated by both pure ones.

\subsection{Long term effects, sales potentials}

Another type of externalities accumulates gradually over time. We assume that advertisement at any point in time has two effects in the future. First, the advertisement efforts have a cumulative effect on the way the total market increases and second, the firm showing more cumulative advertisement efforts gets a larger share of this (potentially) expanded market. In order to introduce these externalities, we need several notations.

Let $h_{t^{\prime}}^{A}=\left(j_{1}^{A}, \ldots, j_{t^{\prime}-1}^{A}\right)$ be the sequence of actions ${ }^{6}$ chosen by player $A$ until stage $t^{\prime} \geq 2$ and let $h_{t^{\prime}}^{B}$ be defined similarly for the other player. Let $\Delta^{m \times n}$ denote the set of real-numbered non-negative $m \times n$-matrices such that all components add up to unity, i.e.,

$$
\Delta^{m \times n}=\left\{z \in \mathbb{R}^{m \times n} \mid z_{i j} \geq 0 \text { for all } i, j, \text { and } \sum_{i j} z_{i j}=1\right\} .
$$

Let matrix $U\left(i^{\prime}, j^{\prime}\right) \in \Delta^{2 \times 2}$ be defined by:

$$
U_{i j}\left(i^{\prime}, j^{\prime}\right)= \begin{cases}1 & \text { if }(i, j)=\left(i^{\prime}, j^{\prime}\right) \\ 0 & \text { otherwise. }\end{cases}
$$

\footnotetext{
${ }^{6}$ References to the second phase actions omitted, firms set their Bertrand equilibrium prices.
} 


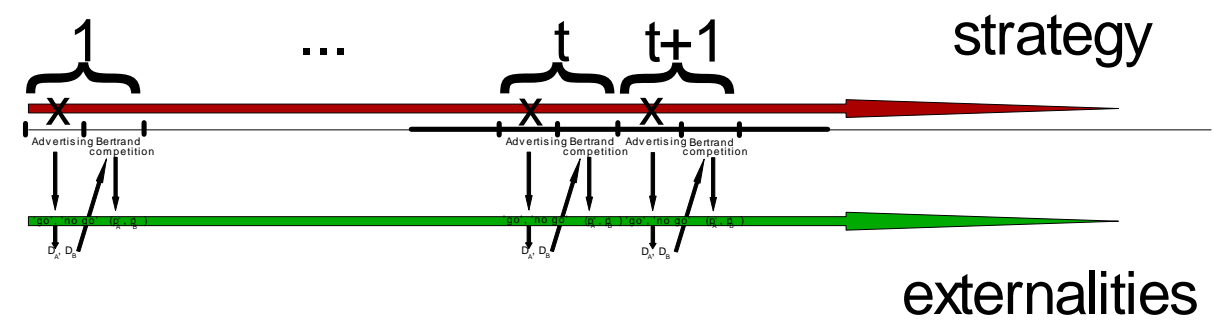

Figure 4: The advertisement strategy is a game plan for the entire timehorizon, the externalities arise because of the decisions along the play. The pricing strategies are Bertrand equilibria, the stage advertisement decisions are determined by the long term strategy.

Then, let $q \geq 0$, and define matrix $\rho_{t} \in \Delta^{2 \times 2}$ recursively for $t \leq t^{\prime}$ by

$$
\begin{aligned}
\rho_{1} & =\widetilde{\rho} \in \Delta^{2 \times 2}, \text { and } \\
\rho_{t} & =\frac{q+t-1}{q+t} \rho_{t-1}+\frac{1}{q+t} U\left(j_{t-1}^{A}, j_{t-1}^{B}\right) .
\end{aligned}
$$

Taking $q \gg 0$ moderates 'early' effects on the stage payoffs. Recall that $j_{t-1}^{A}$ denotes the action chosen by $A$ at stage $t-1$. The interpretation of this matrix is that entry $i j$ of $\rho_{t}$ 'approximates' the relative frequency with which action pair $(i, j)$ was used before stage $t \geq 2$, as it can be shown that

$$
\rho_{t}=\frac{q+1}{q+t} \widetilde{\rho}+\frac{t-1}{q+t} U\left(h_{t}^{A}, h_{t}^{B}\right) .
$$

Here, $U\left(h_{t}^{A}, h_{t}^{B}\right)=\frac{1}{t-1} \sum_{k=2}^{t} U\left(j_{k-1}^{A}, j_{k-1}^{B}\right)$ for all $t \geq 2$. Clearly, the influence of $\widetilde{\rho}$ and $q$ disappears in the long run.

At stage $t \in \mathbb{N}$, the players have chosen action sequences $h_{t}^{A}$ and $h_{t}^{B}$ inducing the matrix $\rho_{t}$. The latter determines the state in which the play is at stage $t .^{7}$ Observe that there exist four possible successor states to any

\footnotetext{
${ }^{7}$ Slightly more formal, we will denote the state at stage $t \in \mathbb{N}$ by $\rho_{t}$ from now on.
} 
state depending on the action pair chosen at stage $t$. Figure 4 visualizes the externalities resulting from the different histories possible.

We assume that some of the parameters used in the previous subsection to formalize the demand functions are indeed given for the maximization problem at hand, i.e., at that stage, but vary over time depending on the advertising behaviors of both firms in the past, i.e., before that stage, in the following manner

$$
(D, a)_{t}=(D, A)\left(\rho_{t}\right)
$$

So, given $\rho_{t}$, the sales potentials ${ }^{8}$ at stage $t$ are given by

$$
S P_{t}^{k}=D_{k}\left(\rho_{t}\right)-a_{1 k}\left(\rho_{t}\right) p_{k}+a_{2 k}\left(\rho_{t}\right) p_{\neg k}, k=A, B .
$$

where all the functions concerned are continuous functions from $\Delta^{2 \times 2}$ to $\mathbb{R}$. The sales potential of a firm at a certain stage is influenced by the firm's own advertisement efforts before, but also by the other player's past advertisement efforts. Own past efforts are always positive ceteris paribus, i.e., the sales potential is always higher if the own advertisement efforts have been higher in the past. Also, the own past efforts have a stronger impact on the firm's sales potential than the other firm's have. This can be formalized into the following set of restrictions:

$$
\left\{\begin{array}{c}
\frac{\partial D_{A}}{\partial\left[\rho_{t}\right]_{11}}, \frac{\partial D_{A}}{\partial\left[\rho_{t}\right]_{12}}, \frac{\partial D_{B}}{\partial\left[\rho_{t}\right]_{11}}, \frac{\partial D_{B}}{\partial\left[\rho_{t}\right]_{21}} \geq 0, \\
\frac{\partial a_{1 A}}{\partial\left[\rho_{t}\right]_{11}}, \frac{\partial a_{1 A}}{\partial\left[\rho_{t}\right]_{12}}, \frac{\partial a_{2 A}}{\partial\left[\rho_{t}\right]_{11}}, \frac{\partial a_{2 A}}{\partial\left[\rho_{t}\right]_{12}} \leq 0, \\
\frac{\partial a_{1 B}}{\partial\left[\rho_{t}\right]_{11}}, \frac{\partial a_{1 B}}{\partial\left[\rho_{t}\right]_{21}}, \frac{\partial a_{2 B}}{\partial\left[\rho_{t}\right]_{11}}, \frac{\partial a_{2 B}}{\partial\left[\rho_{t}\right]_{21}} \leq 0 .
\end{array}\right.
$$

We do not place stronger restrictions on the functions concerned in order to guarantee the highest degree of generality. Sales potentials materialize as demand whenever both firms advertise at the start of the stage game. Otherwise, some potential will evaporate. We now show how the above can be incorporated into an attractive model.

Example 3 Let, for given $\rho_{t}$, the sales potentials be given by (1), where

$$
\begin{aligned}
& D_{A}\left(\rho_{t}\right)=240+80\left(\left[\rho_{t}\right]_{11}+\left[\rho_{t}\right]_{12}\right)\left(3\left[\rho_{t}\right]_{11}+\left[\rho_{t}\right]_{12}+\left[\rho_{t}\right]_{21}\right), \\
& D_{B}\left(\rho_{t}\right)=240+80\left(\left[\rho_{t}\right]_{11}+\left[\rho_{t}\right]_{21}\right)\left(3\left[\rho_{t}\right]_{11}+\left[\rho_{t}\right]_{12}+\left[\rho_{t}\right]_{21}\right), \\
& a_{1 A}\left(\rho_{t}\right)=24-6\left(\left[\rho_{t}\right]_{11}+\left[\rho_{t}\right]_{12}\right), \\
& a_{2 A}\left(\rho_{t}\right)=8-4\left(\left[\rho_{t}\right]_{11}+\left[\rho_{t}\right]_{21}\right), \\
& a_{1 B}\left(\rho_{t}\right)=24-6\left(\left[\rho_{t}\right]_{11}+\left[\rho_{t}\right]_{21}\right), \\
& a_{2 B}\left(\rho_{t}\right)=8-4\left(\left[\rho_{t}\right]_{11}+\left[\rho_{t}\right]_{12}\right) .
\end{aligned}
$$

\footnotetext{
${ }^{8}$ The term market potential was already taken for instance by Ziesemer [1994] or Feichtinger [1982], to name but a few. Ziesemer furthermore uses endogenous changes but these come from diffusion and learning processes as in Amable [1992] rather than advertising decisions.
} 
Advertising raises the common part of the intercepts of the demand functions for both firms in the same manner, i.e., $80\left(3\left[\rho_{t}\right]_{11}+\left[\rho_{t}\right]_{12}+\left[\rho_{t}\right]_{21}\right)$, firm $A$ gets a 'share' of $\left(\left[\rho_{t}\right]_{11}+\left[\rho_{t}\right]_{12}\right)$ of this increase, whereas $B$ gets a 'share' of $\left(\left[\rho_{t}\right]_{11}+\left[\rho_{t}\right]_{21}\right)$. So, the more often $\mathrm{A}$ has advertised in the past the larger its 'share' is, now. The words share are in quotation marks, because they do not sum to unity. Note that there exist externalities as it is for both firms beneficial to coordinate on simultaneous advertising, i.e., keeping $3\left[\rho_{t}\right]_{11}+\left[\rho_{t}\right]_{12}+\left[\rho_{t}\right]_{21}$ constant, the gain with respect to the positive intercept is maximized by having $\left[\rho_{t}\right]_{11}=1$.

Advertising does not only have an impact on the demand functions in the sense that they move shift upward or downward. The parameters indicating the slopes of the demand function in the own price $\left(a_{1 k}\right)$ and the other price $\left(a_{2 k}\right)$ may change as well, hence the associated functions tilt. Note that more intense own past advertisement efforts have been, the less the own demand drops in case of an own price increase; the own demand suffers from increased past advertisement efforts by the other firm.

Having explained how the sales potentials depend on past advertising behavior, we now link the sales potentials and the actual sales in the stage game. First, the sales potentials are given by Eq. (5):

$$
S P_{t}^{k}=D_{k}\left(\rho_{t}\right)-a_{1 k}\left(\rho_{t}\right) p_{k}+a_{2 k}\left(\rho_{t}\right) p_{\neg k}, k=A, B .
$$

Each of the functions is specified above. The actual sales materializing from the sales potentials depend on the current advertisement decisions as follows

$$
\begin{aligned}
x_{A, t}^{1,1} & =1 \cdot S P_{t}^{A} \text { and } x_{B, t}^{1,1}=1 \cdot S P_{t}^{B}, \\
x_{A, t}^{1,2} & =\frac{7}{8} \cdot S P_{t}^{A} \text { and } x_{B, t}^{1,2}=\frac{5}{8} \cdot S P_{t}^{B}, \\
x_{A, t}^{2,1} & =\frac{5}{8} \cdot S P_{t}^{A} \text { and } x_{B, t}^{2,1}=\frac{7}{8} \cdot S P_{t}^{B}, \\
x_{A, t}^{2,2} & =\frac{1}{2} \cdot S P_{t}^{A} \text { and } x_{B, t}^{2,2}=\frac{1}{2} \cdot S P_{t}^{B} .
\end{aligned}
$$

Hence, the total stage game sales are realized if both agents do advertise in the stage game, otherwise some potential is lost, up to half evaporates in case nobody advertises. By Lemma 2 we have the same Bertrand prices in all four cases, and the associated demands are simple multiples as follows:

$$
p_{k}^{*}\left(D^{\prime}, a^{\prime}\right)=p_{k}^{*}(D, a) \text { and } x_{k}^{*}\left(D^{\prime}, a^{\prime}\right)=\gamma_{k} x_{k}^{*}(D, a) .
$$

Please observe that the numerical examples treated in the preceding subsections can be recovered as a special case by setting $\left[\rho_{t}\right]_{22}=1$.

\section{Strategies and rewards}

At stage $t$, both players know the current state and the history of play, i.e., the state visited and actions chosen at stage $u<t$ denoted by $\left(\rho_{u}, j_{u}^{A}, j_{u}^{B}\right)$. 
A strategy prescribes at all stages, for any state and history, a mixed action to be used by a player. The sets of all strategies for $A$ respectively $B$ will be denoted by $\mathcal{X}^{A}$ respectively $\mathcal{X}^{B}$, and $\mathcal{X} \equiv \mathcal{X}^{A} \times \mathcal{X}^{B}$. The payoff to player $k, k=A, B$, at stage $t$, is stochastic and depends on the strategy-pair $(\pi, \sigma) \in \mathcal{X}$; the expected stage payoff is denoted by $R_{t}^{k}(\pi, \sigma)$. In Figure 4 we have visualized the fact that the strategy determines the advertisement decisions in the respective stages and that externalities are caused by these decisions along the play.

The players receive an infinite stream of stage payoffs during the play, and they are assumed to wish to maximize their average rewards. For a given pair of strategies $(\pi, \sigma)$, player $k$ 's average reward, $k=A, B$, is given by $\gamma^{k}(\pi, \sigma)=\liminf _{T \rightarrow \infty} \frac{1}{T} \sum_{t=1}^{T} R_{t}^{k}(\pi, \sigma) ; \gamma(\pi, \sigma) \equiv\left(\gamma^{A}(\pi, \sigma), \gamma^{B}(\pi, \sigma)\right)$.

It may be quite hard to determine the set of feasible (average) rewards $F$, directly. It is not uncommon in the analysis of repeated or stochastic games to limit the scope of strategies on the one hand, and to focus on rewards on the other. Here, we will do both, we focus on rewards from strategies which are pure and jointly convergent. Then, we extend our analysis to obtain more feasible rewards.

A strategy is pure, if at each stage a pure action is chosen, i.e., the action is chosen with probability 1 . The set of pure strategies for player $k$ is $\mathcal{P}^{k}$, and $\mathcal{P} \equiv \mathcal{P}^{A} \times \mathcal{P}^{B}$. The strategy pair $(\pi, \sigma) \in \mathcal{X}$ is jointly convergent if and only if $z^{\pi, \sigma} \in \Delta^{m \times n}$ exists such that for all $\varepsilon>0$ :

$$
\lim \sup _{t \rightarrow \infty} \operatorname{Pr}_{\pi, \sigma}\left[\left|\frac{\#\left\{j_{u}^{A}=i \text { and } j_{u}^{B}=j \mid 1 \leq u \leq t\right\}}{t}-z_{i j}^{\pi, \sigma}\right| \geq \varepsilon\right]=0 \text { for all }(i, j) \in J \text {. }
$$

where $\operatorname{Pr}_{\pi, \sigma}$ denotes the probability under strategy-pair $(\pi, \sigma) . \quad \mathcal{J C}$ denotes the set of jointly-convergent strategy pairs. Under a pair of jointlyconvergent strategies, the relative frequency of each action pair $(i, j) \in J$ converges with probability 1 to $z_{i j}^{\pi, \sigma}$ in the terminology of Billingsley [1986, p.274], i.e., this implies $\lim _{t \rightarrow \infty} E_{\pi, \sigma}\left\{U\left(h_{t}^{A}, h_{t}^{B}\right)\right\}=z^{\pi, \sigma}$. However, this implies also $\lim _{t \rightarrow \infty} E_{\pi, \sigma}\left\{\rho_{t}\right\}=z^{\pi, \sigma}$.

The set of jointly-convergent pure-strategy rewards is given by

$$
P^{\mathcal{J C}} \equiv \operatorname{cl}\left\{\left(x^{1}, x^{2}\right) \in \mathbb{R}^{2} \mid \exists_{(\pi, \sigma) \in \mathcal{P} \cap \mathcal{J C}}:\left(\gamma^{k}(\pi, \sigma), \gamma^{k}(\pi, \sigma)\right)=\left(x^{1}, x^{2}\right)\right\},
$$

where $c l S$ is the closure of the set $S$. For any pair of rewards in this set, we can find a pair of jointly-convergent pure strategies that yield rewards arbitrarily close to the original pair of rewards.

The following result, illustrated in Figure 5, can be found in Joosten et al. [2003] for FD-games. Related ideas were designed for the analysis of repeated games with vanishing actions (cf., Joosten, Peters \& Thuijsman [1995], Joosten [1996, 2005], Schoenmakers et al. [2002], Schoenmakers [2004]). Let $C P^{\mathcal{J C}}$ denote the convex hull of $P^{\mathcal{J C}}$.

Theorem 3 For any FD-game, we have $P^{\mathcal{J C}}=\bigcup_{z \in \Delta^{m \times n}} \varphi(z)$. Moreover, each pair of rewards in $C P^{\mathcal{J C}}$ is feasible. 


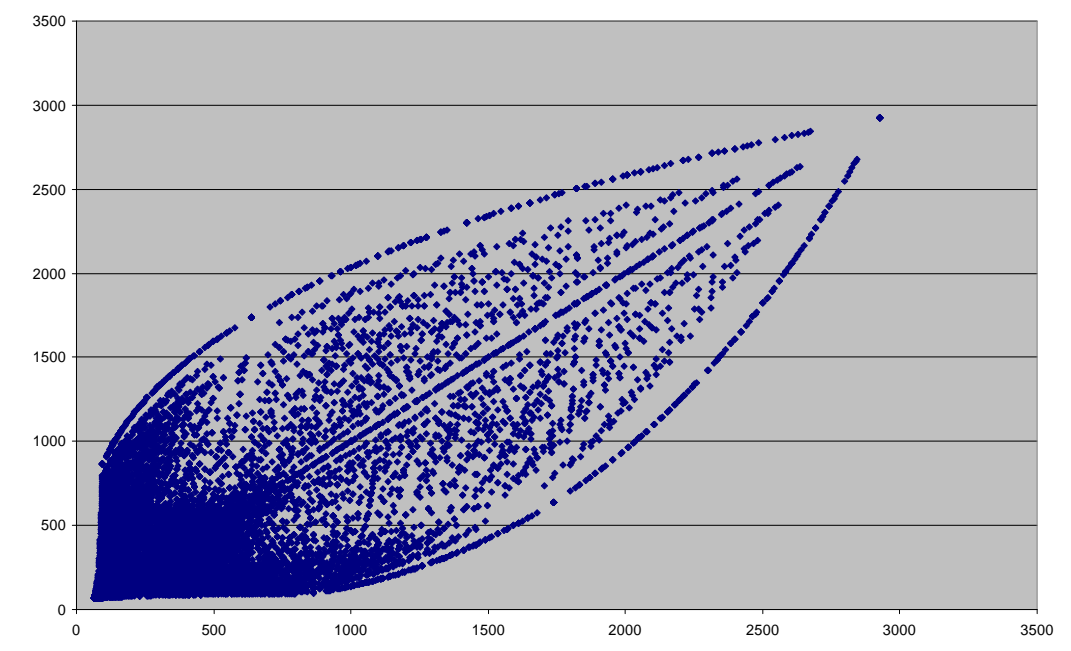

Figure 5: The set of jointly-convergent pure strategy rewards resembles a spearhead. The figure contains holes but the real set is dense. The Paretoefficient equilibrium coincides with both firms always advertising .

From the formulation of Theorem 3 it may not be apparent, but an implication is that $P^{\mathcal{J C}}$ can be visualized rather elegantly. For this purpose, several algorithms have been designed, involving the computation of a pair of feasible rewards for a significant number of 'frequency-matrices' $z \in \Delta^{m \times n}$.

\section{$5 \quad$ Equilibria}

The strategy pair $\left(\pi^{*}, \sigma^{*}\right)$ is an equilibrium, if no player can improve by unilateral deviation, i.e., $\gamma^{A}\left(\pi^{*}, \sigma^{*}\right) \geq \gamma^{A}\left(\pi, \sigma^{*}\right), \gamma^{B}\left(\pi^{*}, \sigma^{*}\right) \geq \gamma^{B}\left(\pi^{*}, \sigma\right)$ for all $\pi \in \mathcal{X}^{A}, \sigma \in \mathcal{X}^{B}$. An equilibrium is called subgame perfect if for each possible state and possible history (even unreached states and histories) the subsequent play corresponds to an equilibrium, i.e., no player can improve by deviating unilaterally from then on.

In the construction of equilibria for repeated games, 'threats' play an important role. A threat specifies the conditions under which one player will punish the other, as well as the subsequent measures. We call $v=$ $\left(v^{A}, v^{B}\right)$ the threat point, where $v^{A}=\min _{\sigma \in \mathcal{X}^{B}} \max _{\pi \in \mathcal{X}^{A}} \gamma^{A}(\pi, \sigma)$, and $v^{B}=\min _{\pi \in \mathcal{X}^{A}} \max _{\sigma \in \mathcal{X}^{B}} \gamma^{B}(\pi, \sigma)$. So, $v^{A}$ is the highest amount $A$ can get if $B$ tries to minimize his average payoffs. Under a pair of individually rational rewards each player receives at least his threat-point reward.

To present the general idea of the result of Joosten et al. [2003] to come, we adopt terms from Hart [1985], Forges [1986] and Aumann \& Maschler [1995]. First, there is a 'master plan' which is followed by each player as long 
as the other does too; then there are 'punishments' which come into effect if a deviation from the master plan occurs. The master plan is a sequence of 'intra-play communications' between the players, the purpose of which is to decide by which equilibrium the play is to continue. The outcome of the communication period is determined by a 'jointly controlled lottery', i.e., at each stage of the communication period the players randomize with equal probability on both actions; at the end of the communication period one sequence of pairs of action choices materializes.

Detection of deviation from the master plan after the communication period is easy as both players use pure actions on the equilibrium path from then on. Deviation during the communication period by using an alternative randomization on the actions is impossible to detect. However, it can be shown that no alternative unilateral randomization yields a higher reward. So, the outcome of the procedure is an equilibrium. For more details, we refer to Joosten et al. [2003]. We restate here the major result which applies to general games with frequency-dependent stage payoffs as well as to JFDgames.

Theorem 4 (Joosten, Brenner $\&$ Witt [2003]) Each pair of rewards in the convex hull of all individually-rational pure-strategy rewards can be supported by an equilibrium. Moreover, each pair of rewards in the convex hull of all pure-strategy rewards giving each player strictly more than the threat-point reward, can be supported by a subgame-perfect equilibrium.

The following is visualized in Figure 6 and illustrated in Example 4.

Corollary 5 Let $E^{\prime}=\left\{(x, y) \in P^{\mathcal{J C}} \mid(x, y) \geq v\right\}$ be the set of all individually rational jointly-convergent pure-strategy rewards. Then, each pair of rewards in the convex hull of $E^{\prime}$ can be supported by an equilibrium. Moreover, all rewards in $E^{\prime}$ giving $A$ strictly more than $v^{A}$ and $B$ strictly more than $v^{B}$ can be supported by a subgame-perfect equilibrium.

In determining the set of jointly convergent pure strategy rewards and the sets depending on it, we took Bertrand equilibrium pricing as behavior for the stage game. To execute a 'threat' this need no longer hold, i.e., a player may very well punish using a low price at each stage in addition to a long term advertisement strategy in order to minimize his opponent's rewards. The following may serve as an example.

Example 4 It is quite difficult to find a threat point in general. Instead, we will establish an upper bound for the threat point rewards. This implies that all rewards above this upper bound can be supported by a subgame perfect equilibrium.

We assume that firm $A$ is to be punished by firm $B$ and firm $B$ simply sets 
a price and takes a long term advertising decision. Let therefore, $p_{B}=4.95$ and $\rho_{11}=\rho_{21}=0$, then the long-run average profit of firm $A$ is

$$
\begin{aligned}
& \pi^{A}\left(\rho_{12}, p_{A}\right)= \\
& \left(\frac{1}{2}+\frac{3}{8} \rho_{12}\right)\left(279.6+80 \rho_{12}^{2}-\left(24-6 \rho_{12}\right) p_{A}\right)\left(p_{A}-3\right)-200-200 \rho_{12},
\end{aligned}
$$

which can be motivated as follows. If firm $A$ advertises it has stage profits

$$
\left(\frac{7}{8}\right)\left(279.6+80 \rho_{12}^{2}-\left(24-6 \rho_{12}\right) p_{A}\right)\left(p_{A}-3\right)-400,
$$

whereas if it does not, it obtains

$$
\left(\frac{1}{2}\right)\left(279.6+80 \rho_{12}^{2}-\left(24-6 \rho_{12}\right) p_{A}\right)\left(p_{A}-3\right)-200 .
$$

The long-run frequency of the first event is $\rho_{12}$, the frequency of the second one is $\rho_{22}=1-\rho_{12}$. Note that in the first event the costs are 200 higher because the agent has to pay for the advertising efforts. Multiplying the events' profits with their relative frequencies, yields the average long run profits.

Note that if firm $A$ wishes to maximize the long-run average profits, it may use both strategic variables $\rho_{12}, p_{A}$. We determine therefore the first derivatives of the long-run average profit function.

$$
\begin{aligned}
\frac{\partial \pi^{A}}{\partial p_{A}}=\left(\frac{1}{2}+\right. & \left.\frac{3}{8} \rho_{12}\right)\left(279.6+80 \rho_{12}^{2}-2\left(24-6 \rho_{12}\right) p_{A}+3\left(24-6 \rho_{12}\right)\right) \\
\frac{\partial \pi^{A}}{\partial \rho_{12}}= & \frac{3}{8}\left(279.6+80 \rho_{12}^{2}-\left(24-6 \rho_{12}\right) p_{A}\right)\left(p_{A}-3\right) \\
& +\left(\frac{7}{8} \rho_{12}+\frac{1}{2}\left(1-\rho_{12}\right)\right)\left(160 \rho_{12}+6 p_{A}\right)\left(p_{A}-3\right)-200
\end{aligned}
$$

Then, $\frac{\partial \pi^{A}}{\partial p_{A}}=0$ is equivalent to $p_{A}=\frac{3}{2}+\frac{279.6+80 \rho_{12}^{2}}{48-12 \rho_{12}}$. Inserting the latter in the formula for $\frac{\partial \pi^{A}}{\partial \rho_{12}}$ and solving for $\frac{\partial \pi^{A}}{\partial \rho_{12}}=0$ yields five roots, two with imaginary parts and three outside the range $[0,1]$. So, an interior solution to the maximization problem is not available. Taking the price of firm $A$ to the extremes does not make sense as both minimum and maximum prices yield losses. Therefore, we must consider either $\rho_{12}=0$ or $\rho_{12}=1$. A simple comparison yields that $\rho_{12}=1$ yields highest profits 734.96 , based on computations $p_{A}=11.489$ and $x_{A}=133.70$.

Note furthermore that firm $B$ 's profit is

$$
\frac{5}{8}(167.16)(4.95-3)-200=3.7263 .
$$


So, firm $B$ can indeed keep firm $A$ 's long term stage payoffs at at most 734.96 without the fear of going bankrupt, which lends considerable credibility to this threat. This implies that firm A's rewards can be kept below 734.96 as well. Hence, we have shown that an upper bound for the threat point is

$$
\bar{v}=(734.96,734.96) .
$$

This in turn implies that all rewards yielding more than 734.96 for both firms can be supported by an equilibrium (a subgame perfect equilibrium) using threats (see Figure 6 for an illustration).

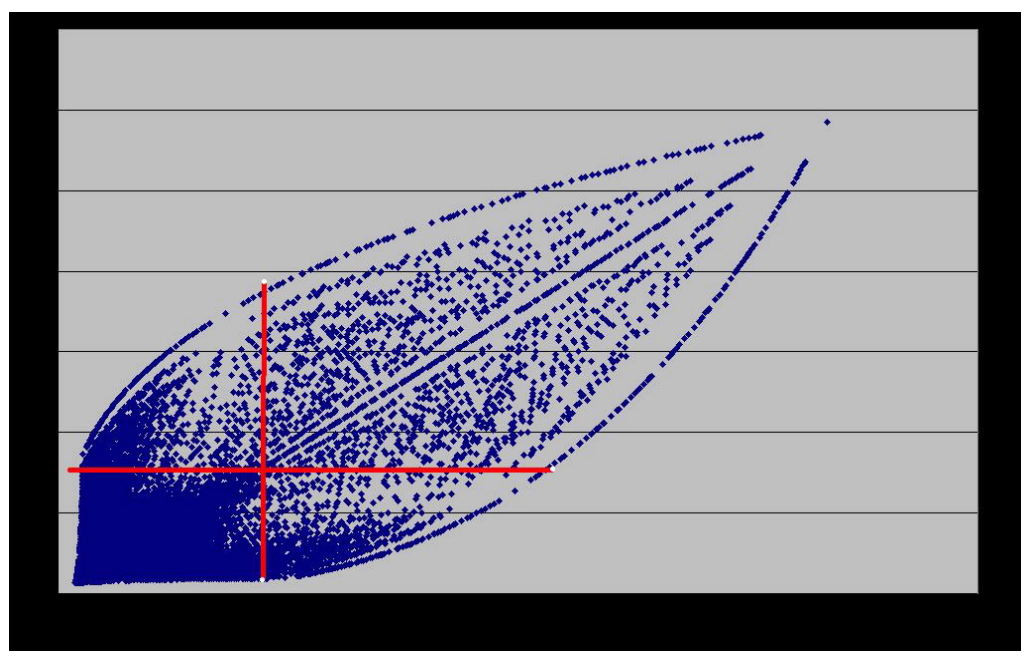

Figure 6: All rewards in $P^{\mathcal{J C}}$ located to the 'north-east' of the two red lines can be supported by a subgame perfect equilibrium.

\section{Conclusion}

We have formulated a new dynamic model of advertising in very general terms. A broad variety of long and short term externalities can be modeled by altering parameters. Long run advertising changes the height and shape of each firm's sales potential (function) in a rather gradual fashion. From the sales potentials, the current demands are derived and the latter depend on the current advertising decisions. If both firms advertise at that stage, then their potentials will become their immediate demands, otherwise some potential is lost and will not materialize in current demand.

Our analysis has considerable similarities with methods standard in the study of repeated games. We regard this a major strength of the approach, large sets of equilibria are relatively easily found and the variety of mathematical equations allowed is almost limitless as we only need a weak continuity assumption to validate them. Admittedly, a slight drawback of the 
approach is the familiar one connected to Folk Theorems, namely that there are far too many equilibria..$^{9}$ For this purpose, additional selection criteria, such as Pareto-efficiency ${ }^{10}$, might prove very useful. Equilibrium selection is however, not a theme of this paper.

We have analyzed one example of the model rather completely. Questions may arise about the robustness of this example with respect to alternative specifications. We have performed sensitivity analysis on all parameters. The qualitative features of the set of jointly convergent pure strategy rewards, so important for our analysis, are remarkably unaffected by even rather large changes. For one-parameter changes we mostly observed shifts and expansions of the basic 'spearhead', but the shape of $P^{\mathcal{J C}}$ remained essentially the same. We did not perform a complete sensitivity analysis on combinations of parameters, which is an arduous task.

The parameter with the most dramatic effects turned out to be the advertisement fee. For high values, only one equilibrium exists namely never to advertise at all. For low advertisement costs, the unique Pareto efficient equilibrium is to advertise always, and a continuum of equilibria exists which are not Pareto efficient. For intermediate costs, a continuum of equilibria exists and a continuum of Pareto efficient equilibria exists. The latter range of costs is extremely small relatively speaking.

An illustration of a case where the advertisement costs are considerably above the value used throughout this paper is given in Figure 7. In the tail of the fish shape, those rewards are to be found which result from strategies which do not yield enough long-run externalities for the sales potentials to expand sufficiently to justify incurring the advertisement fees. We have established an upper bound for the threat point of approximately zero, hence each reward in the positive orthant can be supported by a subgame perfect equilibrium. Note that this implies that although the shape of $P^{\mathcal{J C}}$ changes considerably, the shape of the set of equilibria is not fundamentally different from its shape for lower values of the advertisement fees.

In addition to a certain degree of robustness regarding parameters, we may also report a robustness as to the specifications of the market in the stage games. In Joosten [2009], we investigated long term advertising with numbers plausible from an assumption of Cournot competition. Further research must bring a more complete picture as to what to expect under different assumptions regarding competition in the stage games.

We have made concessions to reality to obtain results. We modeled advertising as an either-or decision, not taking into account that various

\footnotetext{
${ }^{9}$ Opinions in the profession vary widely and wildly. Gintis [2001] for instance, dismisses Folk Theorems as 'anything goes results'. On the other hand, Osborne \& Rubinstein [1994] point out that Folk Theorems may yield considerable Pareto improvements compared to infinetely repeated one-shot Nash equilibria.

${ }^{10}$ Alternative selection criteria are advocated in e.g.,. Schelling [1960], Sugden [1995], Janssen [1998, 2001]) or Güth et al. [1992].
} 


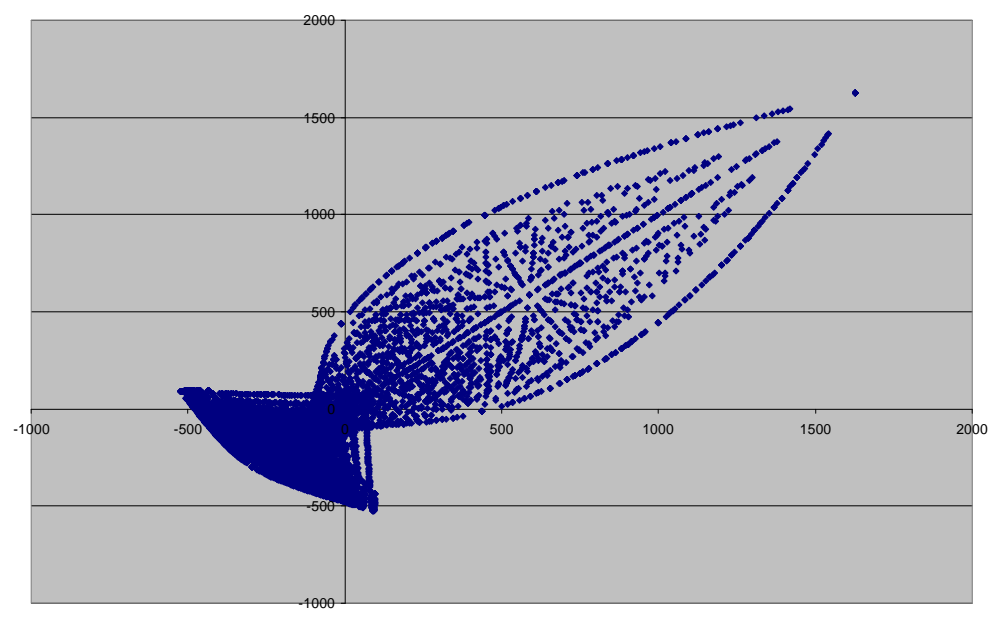

Figure 7: For increasing advertisement costs, $P^{\mathcal{J C}}$ changes gradually into a fish shape. Here, the advertisement costs are equal to 1500.

budgets might be attributed to it. However, our model is easily generalized as any one-period budget in a finite range with a smallest monetary unit can be modeled as a separate action. We refrained from doing so as the added notational burdens seem hardly justified by additional insights.

Vidale \& Wolfe [1957] described the interaction of advertising and sales using a simple differential equation in terms of three parameters, the sales decay constant, the saturation level, and the response constant. Some of the phenomena these parameters are meant to capture, are present in our model, albeit implicitly. Further research must reveal whether such empirical findings can be approximated to a higher degree. The building blocks of our model are easily adapted to accommodate input from empirics.

The class of $(\mathrm{J}) \mathrm{FD}$-games is rather new and the tools for analysis are far from complete. Some results beyond the framework of this paper have been established for environmental problems (Joosten [2004]) and so-called Small Fish Wars (Joosten [2007a,b,c, 2010], Joosten \& Meijboom [2010]). Extending the model to allow an $n$-firm advertisement game is high on the agenda. ${ }^{11}$ Large parts of the approach, most importantly Theorem 4 , seem generalizable, but a comprehensive formal generalization is still pending.

A crucial step in our approach is finding all jointly-convergent purestrategy rewards, another one is determining the threat point. For the first step continuity of the functions determining the average payoffs on the relevant domains of the stochastic variables involved, suffices. Unfortunately, there exists no general theory on (finding) threat points in FD-games, yet.

\footnotetext{
${ }^{11}$ Joosten [2010] also examines 3-person Fish Wars.
} 


\section{Appendix}

Proof of Lemma 1. Let $(D, a)$ be given and to economize on notations, we omit references to $(D, a)$ as long as it seems harmless to do so. The maximization problems formulated in (4) yield the following first-order condition for a supremum :

$$
0=D_{k}-2 a_{1 k} p_{k}+a_{2 k} p_{k}+c_{k} a_{1 k} \text { for } k=A, B \text {. }
$$

The second-order conditions for a supremum are therefore easily fulfilled as

$$
-2 a_{1 k}<0 \text { for } k=A, B .
$$

From the first-order conditions we derive the following reaction curves (omitting references to the parameters):

$$
p_{k}\left(p_{\neg k}\right)=\frac{D_{k}+a_{2 k} p_{\neg k}+c_{k} a_{1 k}}{2 a_{1 k}}, k=A, B .
$$

Then, using the idea that a Bertrand equilibrium involves price strategies which mutual best replies, we may state that the solution obeys the fixed point criterion $p_{A}^{*}=p_{A}\left(p_{B}\left(p_{A}^{*}\right)\right)$ yielding

$$
\begin{aligned}
p_{A}^{*} & =p_{A}\left(p_{B}\left(p_{A}^{*}\right)\right)=\frac{D_{A}+a_{2 A} p_{B}\left(p_{A}^{*}\right)+c_{A} a_{1 A}}{2 a_{1 A}} \\
& =\frac{D_{A}+a_{2 A}\left(\frac{D_{B}+a_{2 B} p_{A}^{*}+c_{B} a_{1 B}}{2 a_{1 B}}\right)+c_{A} a_{1 A}}{2 a_{1 A}} .
\end{aligned}
$$

Pre-multiplying the first and last parts of the above with $4 a_{1 A} a_{1 B}$ yields

$$
\begin{aligned}
4 a_{1 A} a_{1 B} p_{A}^{*} & =2 a_{1 B} D_{A}+a_{2 A}\left(D_{B}+a_{2 B} p_{A}^{*}+c_{B} a_{1 B}\right)+2 a_{1 B} c_{A} a_{1 A} \\
& =a_{2 A} a_{2 B} p_{A}^{*}+2 a_{1 B}\left(D_{A}+c_{A} a_{1 A}\right)+a_{2 A}\left(D_{B}+c_{B} a_{1 B}\right) .
\end{aligned}
$$

Hence, $\left(4 a_{1 A} a_{1 B}-a_{2 A} a_{2 B}\right) p_{A}^{*}=2 a_{1 B}\left(D_{A}+c_{A} a_{1 A}\right)+a_{2 A}\left(D_{B}+c_{B} a_{1 B}\right)$. This gives the statement of the lemma for $A$ under (2). The statement for $B$ can be verified similarly.

Proof of Lemma 2. Since

$$
\begin{aligned}
p_{k}^{*}\left(D^{\prime}, a^{\prime}\right) & =\frac{2 \gamma_{\neg k} a_{1 \neg k}\left(\gamma_{k} D_{k}+c_{k} \gamma_{k} a_{1 k}\right)+\gamma_{k} a_{2 k}\left(\gamma_{\neg k} D_{\neg k}+c_{\neg k} \gamma_{\neg k} a_{1 \neg k}\right)}{4 \gamma_{A} a_{1 A} \gamma_{B} a_{1 B}-\gamma_{A} a_{2 A} \gamma_{B} a_{2 B}} \\
& =\frac{\gamma_{A} \gamma_{B}\left(2 a_{1 \neg k}\left(D_{k}+c_{k} a_{1 k}\right)+a_{2 k}\left(D_{\neg k}+c_{\neg k} a_{1 \neg k}\right)\right)}{\gamma_{A} \gamma_{B}\left(4 a_{1 A} a_{1 B}-a_{2 A} a_{2 B}\right)} \\
& =\frac{2 a_{1 \neg k}\left(D_{k}+c_{k} a_{1 k}\right)+a_{2 k}\left(D_{\neg k}+c_{\neg k} a_{1 \neg k}\right)}{4 a_{1 A} a_{1 B}-a_{2 A} a_{2 B}}=p_{k}^{*}(D, a),
\end{aligned}
$$


and

$$
\begin{aligned}
x_{k}^{*}\left(D^{\prime}, a^{\prime}\right) & =\frac{1}{2}\left(\gamma_{k} D_{k}-c_{k} \gamma_{k} a_{1 k}\right)+\frac{\gamma_{k} a_{1 k} \gamma_{k} a_{2 k}\left(\gamma_{\neg k} D_{\neg k}+c_{\neg k} \gamma_{\neg k} a_{1 \neg k}\right)}{4 \gamma_{A} a_{1 A} \gamma_{B} a_{1 B}-\gamma_{A} a_{2 A} \gamma_{B} a_{2 B}} \\
& =\frac{1}{2}\left(\gamma_{k} D_{k}-c_{k} \gamma_{k} a_{1 k}\right)+\frac{\gamma_{k} a_{1 k} a_{2 k}\left(D_{\neg k}+c_{\neg k} a_{1 \neg k}\right)}{4 a_{1 A} a_{1 B}-a_{2 A} a_{2 B}} \\
& =\gamma_{k}\left(\frac{1}{2}\left(D_{k}-c_{k} a_{1 k}\right)+\frac{a_{1 k} a_{2 k}\left(D D_{\neg k}+c_{\neg k} a_{1 \neg k}\right)}{4 a_{1 A} a_{1 B}-a_{2 A} a_{2 B}}\right) \\
& =\gamma_{k} x_{k}^{*}(D, a),
\end{aligned}
$$

the statement of the lemma follows immediately.

\section{References}

Amable B, 1992, Competition among techniques in the presence of increasing returns to scale, J Evol Econ 2, 147-158.

Amir R, 2003, Stochastic games in economics and related fields: an overview, in: "Stochastic Games and Applications" (A Neyman \& S Sorin, eds.), NATO Advanced Study Institute, Series D, Kluwer, Dordrecht, pp. 455-470.

Aumann RJ, \& M Maschler, 1995, "Repeated Games of Incomplete Information", MIT Press, Cambridge, MA.

Billingsley P, 1986, "Probability and Measure", John Wiley \& Sons, New York, NY.

Brenner T, \& U Witt, 2003, Melioration learning in games with constant and frequency-dependent payoffs, J Econ Behav Organ 50, 429-448.

Cellini R \& L Lambertini, 2003, Advertising with spillover effects in a differential oligopoly game with differentiated goods, Eur J Oper Res 11, 409-423.

Cellini R, L Lambertini \& A Mantovani, 2008, Persuasive advertising under Bertrand competition: A differential game, OR Letters 36, 381-384.

Darby M, \& E Karni, 1973, Free competition and the optimal amount of fraud, J Law \& Econ 16, 67-88.

Dockner E, S Jørgensen, N Van Long, \& G Sorger, 2000, "Differential Games in Economics and Management Science", Cambridge University Press, Cambridge, UK.

Dorfman R, \& PO Steiner, 1954, Optimal advertising and optimal quality, Amer Econ Rev 44, 826-836.

Engwerda J, 2005, "LQ Dynamic Optimization and Differential Games", John Wiley \& Sons Ltd., Chichester, UK.

Feichtinger G, 1982, Optimal pricing in a diffusion model with concave price-dependent market potential, OR Letters 1, 236-240.

Feichtinger G, RF Hartl, \& SP Sethi, 1994, Dynamic optimal control models in advertising: recent developments, Managem Science 40, 195-226. 
Fershtman C, 1984, Goodwill and market shares in oligopoly, Economica 51, 271-281.

Forges F, 1986, An approach to communication equilibria, Econometrica 54, 1375-1385.

Friedman JW, 1983, Advertising and oligopolistic equilibrium, Bell J Econ 14, 464-473.

Gintis H, 2001, "Game Theory Evolving", Princeton University Press, Princeton, NJ.

Güth W, W Leininger \& G Stephan, 1991, On supergames and folk theorems - A conceptual discussion, in: "Game Equilibrium Models II, Methods, Morals, and Markets" (R Selten, ed), Springer, Berlin, pp. 56-70.

Hart S, 1985, Nonzero-sum two-person repeated games with incomplete information, Math Oper Res 10, 117-153.

Janssen MCW, 1998, Focal points, in: "New Palgrave Dictionary of Economics and Law", Vol II (P Newman, ed.), MacMillan, London, pp. 150-155. Janssen MCW, 2001, Rationalizing focal points, Theory and Decision 50, 119-148.

Joosten R, 1996, "Dynamics, Equilibria, and Values", Ph.D.-thesis, Maastricht University.

Joosten R, 2004, Strategic interaction and externalities: FD-games and pollution, Papers Econ $\mathscr{E}$ Evol \# 0417, Max Planck Institute of Economics, Jena.

Joosten R, 2005, A note on repeated games with vanishing actions, Int Game Theory Rev 7, 107-115.

Joosten R, 2007a, Small Fish Wars: A new class of dynamic fisherymanagement games, ICFAI J Managerial Economics 5, 17-30.

Joosten R, 2007b, Small Fish Wars and an authority, in: "The Rules of the Game: Institutions, Law, and Economics" (A. Prinz, A.E. Steenge \& J. Schmidt, eds.), LIT-Verlag, Muenster, pp. 131-162.

Joosten R, 2007c, Patience, Fish Wars, rarity value and Allee effects, $P a$ pers Econ 83 Evol \# 0724, Max Planck Institute of Economics, Jena.

Joosten R, 2009, Strategic advertisement with externalities: a new dynamic approach, in: "Modeling, Computation and Optimization" (SK Neogy, AK Das \& RB Bapat, eds.), Vol. 6 of the ISI Platinum Jubilee Series, World Scientific Publishing Co., Singapore, pp. 21-43.

Joosten R, 2010, Strong and weak rarity value in Small Fish Wars, submitted to Ecological Economics.

Joosten R, T Brenner \& U Witt, 2003, Games with frequency-dependent stage payoffs, Int $J$ Game Theory 31, 609-620.

Joosten R \& R Meijboom, 2010, Stochastic games with endogenous transition probabilities, research memorandum.

Joosten R, H Peters \& F Thuijsman, 1995, Unlearning by not doing: repeated games with vanishing actions, Games Econ Behav 9, 1-7. 
Jørgensen S, \& G Zaccour, 2004, "Differential Games in Marketing", Kluwer Academic Publishers, Dordrecht.

Kydland F, 1977, Equilibrium solutions in dynamic dominant-player models, J Econ Theory 15, 307-324.

Lambertini L, 2005, Advertising in a dynamics spatial monopoly, Eur $J$ Oper Res 16, 547-556.

Levhari D \& LJ Mirman, 1980, The great fish war: An example using a dynamic Cournot-Nash solution, Bell J Econ 11, 322-334.

Nash J, 1951, Noncooperative games, Ann Math 54, 284-295.

Nelson P, 1970, Information and consumer behavior, J Pol Econ 78, 311329 .

Nerlove M \& KJ Arrow, 1962, Optimal advertising policy under dynamic conditions, Economica 29, 129-142.

Osborne M \& A Rubinstein, 1994, "A Course in Game Theory", MIT Press, Cambridge, MA.

Prescott E, 1973, Market structure and monopoly profits: a dynamic theory, J Econ Theory 6, 546-557.

Schelling TC, 1960, "The Strategy of Conflict", Harvard University Press, Cambridge, MA.

Schoenmakers GM, 2004, "The Profit of Skills in Repeated and Stochastic Games", Ph-D Thesis Maastricht University, ISBN 9090184473.

Schoenmakers G, J Flesch \& F Thuijsman, 2002, Coordination games with vanishing actions, Int Game Theory Rev 4, 119-126.

Schmalensee R, 1978, A model of advertising and product quality, J Pol Economy 86, 485-503.

Sethi SP, 1977, Dynamic optimal control models in advertising: a survey, SIAM Rev 19, 685-725.

Shapley L, 1953, Stochastic games, Proceedings of the National Academy of Sciences U.S.A. 39, 1095-1100.

Sugden R, 1995, A theory of focal points, Economic Journal 105, 533-550.

Vidale ML, \& HB Wolfe, 1957, An operations-research study of sales response to advertising, Operations Research 5, 370-381.

Waugh FV, 1959, Needed research on the effectiveness of farm products promotions, J Farm Econ 41, 364-376.

Ziesemer T, 1994, Dynamic oligopolistic pricing with endogenous change in market structure and market potential in an epidemic diffusion model, in: "The Economics of Growth and Technical Change: Technologies, Nations, Agents" (G Silverberg \& L Soete, eds.), Edward Elgar, Aldershot, UK, pp. 217-239. 